\title{
Erratum to: Diabetes self-management among Arab Americans: patient and provider perspectives
}

Heather Fritz ${ }^{1}$, Rosanne DiZazzo-Miller ${ }^{2 *}$, Elizabeth A. Bertran ${ }^{3}$, Fredrick D. Pociask ${ }^{2}$, Sandra Tarakji ${ }^{4}$, Judith Arnetz ${ }^{5}$, Catherine L. Lysack ${ }^{1}$ and Linda A. Jaber ${ }^{3}$

Unfortunately, the original version of this article [1] contained an error. The acknowledgements section was included incorrectly and should have acknowledged The Martha Schnebly Endowed Research Fund.

\begin{abstract}
Author details
${ }^{1}$ Institute of Gerontology, Wayne State University, 87 East Ferry Street, 226 Knapp Building, Detroit, Ml 48202, USA. ${ }^{2}$ Department of Health Care Sciences, Wayne State University, 259 Mack Ave, Detroit, MI 48201, USA. ${ }^{3}$ Department of Pharamacy Practice, Wayne State University, 259 Mack Ave, Detroit, Ml 48201, USA. ${ }^{4}$ Department of Family Medicine and Public Health Sciences, Wayne State University, Detroit, MI 48202, USA. ${ }^{5}$ Department of Family Medicine, Michigan State University, 788 Service Rd., B103 Clinical Center, East Lansing, Ml 48824, USA.
\end{abstract}

Received: 31 August 2016 Accepted: 1 September 2016

Published online: 20 September 2016

\section{Reference}

1. Fritz et al. Diabetes self-management among Arab Americans: patient and provider perspectives. BMC International Health and Human Rights (2016) 16:22.

\footnotetext{
* Correspondence: ar7975@wayne.edu

${ }^{2}$ Department of Health Care Sciences, Wayne State University, 259 Mack Ave, Detroit, MI 48201, USA
} 